\title{
Fatores que prevalecem ao esgotamento profissional em professores
}

\author{
Adelson Fernandes da Silva ${ }^{a}$, Maria de Fatima de Matos Maia ${ }^{a}$, Celina Aparecida Gonçalves Limaa \\ Izulina Torres Guedes ${ }^{a}$, Karoline Costa Pedreira ${ }^{a}$, Diego Augusto Santos Silva ${ }^{b}$, \\ Edio Luiz Petroski ${ }^{b}$
}

\author{
aUniversidade Estadual de Montes Claros - Unimontes, Montes Claros, MG, Brasil.

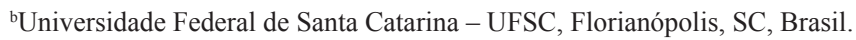

\begin{abstract}
Resumo: Introdução: A síndrome de burnout (SB) é uma reação ao estresse excessivo relacionado ao trabalho. Objetivo: Verificar a prevalência e os fatores associados à síndrome do esgotamento profissional (SEP) nos professores da rede pública dos Ensinos Infantil, Fundamental e Médio. Método: Para este estudo transversal, 462 professores das cidades de Januária, Itacarambi, Manga, São Francisco e Pedras de Maria da Cruz foram entrevistados. Utilizou-se como instrumento o Questionário Preliminar de Identificação do Burnout, que classifica o sujeito em "esgotado" e "não esgotado". Os fatores associados investigados foram: sexo, modalidade de ensino, tempo na docência, se trabalha em outra escola pública, vínculo empregatício, se está satisfeito com a remuneração, jornada semanal na docência e se possui algum tipo de doença. Resultados: Dos professores investigados, $24 \%$ estavam na fase 3 - estágio em que começa a se instalar a SEP - e 4,7\% dos professores estavam na fase 4 - estágio mais crítico da síndrome. A SEP esteve associada à baixa remuneração, à dedicação à carreira de docente e ao tempo de trabalho de um a 11 anos ou mais. Conclusão: Pode-se concluir que a SEP é altamente prevalente entre os professores efetivos e efetivados do ensino público e gratuito.
\end{abstract}

Palavras-chave: Esgotamento Profissional, Professores, Estresse Psicológico.

\section{Prevailing factors causing professional burnout in teachers}

\begin{abstract}
Introduction: Burnout Syndrome (BS) consists in a reaction to excessive work-related stress. Objective: To check the prevalence and associated factors of BS in kindergarten, primary and secondary public school teachers of. Method: A cross-sectional study was carried out; 462 teachers from the cities of Januária, Itacarambi, Manga, São Francisco and Pedras de Maria da Cruz were interviewed. The Preliminary Questionnaire for Burnout identification was the instrument used to classify individuals into "exhausted" and "not exhausted". The associated factors were gender, type of education, time experience with teaching, employment in other public schools, employment bond, satisfaction with the income, weekly teaching hours, and presence of diseases. Results: Among the teachers surveyed, $24 \%$ were in stage 3 , the point when BS begins; and $4.7 \%$ were in stage 4 , the most critical stage of the syndrome. The BS was associated with low pay, dedication to the teaching career and time experience with teaching from one to 11 years or more. Conclusion: That the BS is highly prevalent among permanent and hired teachers of the public and free education network.
\end{abstract}

Keywords: Burnout Professional, Teachers, Stress Psychological. 


\section{Introdução}

A síndrome de burnout (SB) é um fenômeno psicossocial que surge como resposta a estressores crônicos no trabalho, em profissionais que lidam com clientes de forma emocional, direta e constante (GIL-MONTE, 2005; MASLACH, 2003; MASLACH; SCHAUFELI; LEITER, 2001). A SB é reconhecida mundialmente como um dos grandes problemas psicossociais que afetam a qualidade de vida de profissionais de diversas áreas, principalmente daquelas que envolvem cuidados com saúde, educação e serviços humanos, gerando uma importante questão ocupacional e social (SOUSA; MENDONÇA, 2009). No Brasil, desde 1999, a SB é reconhecida como doença profissional, pelo Decreto-Lei no 6.042/07, da Previdência Social, incluída na lista B do grupo V, da Classificação Internacional de Doenças (CID-10).

A síndrome do esgotamento profissional (SEP), ou síndrome de burnout (SB) (MASLACH; JACKSON, 1986), é um termo que tem se destacado no trabalho docente nos últimos anos. A categoria docente tem sido alvo de inúmeros estressores psicossociais presentes no contexto de trabalho. Segundo Both (2011), o profissional da educação desenvolve uma das atividades mais importantes e estressantes da sociedade, em que se requer desprendimento de tempo com a formação do estudante e o comprometimento com as tarefas de preparação das aulas.

Além de ministrar as aulas, o docente deve fazer, concomitantemente, os trabalhos administrativos, planejar as suas atividades letivas, reciclar-se, orientar os alunos e o atendimento aos pais. Deve, ainda, organizar atividades extraescolares, participar de reuniōes de coordenação, seminários, conselhos de classe, preenchimento de relatórios individuais relativos às dificuldades de aprendizagem dos alunos e, muitas vezes, cuidar também do patrimônio, material, recreios e locais de refeiçôes (NACARATO; VARANI; CARVALHO, 2000). Outros fatores estressores que os professores enfrentam na atualidade é o uso de drogas ilícitas no ambiente escolar, principalmente no turno noturno, o desinteresse dos alunos, o uso constante de celulares na sala de aula, bem como o uso indevido das redes sociais na internet, nos horários de aula, violência entre alunos e violências verbal e física de alunos com professores. Problemas motivacionais e comportamentais dos alunos são apontados como agentes estressores significativos e/ou psicológicos em professores (SILVEIRA et al., 2014).

Embora o estresse e o burnout no ensino ocorram certamente há muito tempo entre os professores, o seu reconhecimento como uma importante questão de saúde pública tem sido mais explícito nos últimos anos, em razáo do cenário educacional da atualidade, em que professores são submetidos a diversos fatores que refletem negativamente na profissão (BATISTA et al., 2010). Verifica-se que os resultados encontrados em investigaçôes realizadas por pesquisadores nos últimos anos atestam para a gravidade de burnout nesses profissionais de ensino, em que se enquadra essa categoria profissional, identificada como de alto risco (FARBER, 1991; HEUS; DIEKSTRA, 1999; KOVESS-MASFÉTY et al., 2006).

O cenário educativo brasileiro apresenta um quadro bastante problemático no que se refere ao trabalho do professor, pois as tarefas de alto nível são transformadas em rotinas e há menos tempo para executar o trabalho, atualização profissional, lazer e convívio social, bem como são escassas as oportunidades de trabalho criativo (CARLOTTO, 2011). As mudanças do papel do professor na sociedade contribuem com implicaçôes negativas, pois têm sido associadas a aposentadorias precoces, absenteísmo, licença médica e rotatividade de trabalhadores (SEGURA, 2014).

Tendo em vista que programas de prevenção a doenças psicossociais poderão ser desenvolvidos, o objetivo dessa pesquisa foi verificar a prevalência da SB em professores da rede pública dos Ensinos Infantil, Fundamental e Médio e analisar a associação entre SB e fatores associados (sexo, modalidade de ensino, tempo na área educacional, situação atual no emprego, satisfação com a remuneração, jornada semanal de trabalho e doença) em algumas cidades do Sudeste do Brasil.

\section{Método}

Esta pesquisa caracteriza-se como estudo descritivo, quantitativo e de corte transversal, realizado com uma amostra representativa de professores pertencentes às escolas da jurisdição da Superintendência Estadual de Ensino (SEE) de Januária, em Minas Gerais.

A população do estudo foi composta de 3.260 professores da rede pública estadual, da SEE. Para o cálculo do tamanho amostral, adotou-se prevalência desconhecida para desfecho (igual a 50\%), erro tolerável de cinco pontos percentuais, nível de confiança de $95 \%$, efeito de delineamento de 1,5, acrescentando $15 \%$ para possíveis perdas e recusas. Assim, estimou-se que seria necessário coletar informações de 457 professores. Em razão das características do processo amostral por conglomerado, participaram da amostra 462 professores. Destes, 122 $(26,4 \%)$ eram do sexo masculino e $340(73,6 \%)$, do 
feminino. A idade dos indivíduos foi de 20 a 66 anos $(36,27 \pm 8,52)$.

Os professores participaram da pesquisa de forma aleatória. Na cidade de Januária, duas acadêmicas do curso de Educação Física, da iniciação científica, visitaram as escolas públicas e aplicaram os questionários a todos os professores que aceitaram participar da pesquisa e assinaram o termo de consentimento livre e esclarecido, CAAE: 08226512.5.0000.5146, aprovado em 23 de outubro de 2012. Os professores das cidades de Itacarambi, Manga, São Francisco e Pedras de Maria da Cruz participaram da pesquisa por conglomerado em um encontro educacional que aconteceu na Universidade Estadual de Montes Claros, no Campus Januária, realizado pela SEE.

Os critérios de inclusão/exclusão observados foram: ser professor na rede pública de ensino, estar no momento em sala de aula e náo exercer cargo de gestão. Para a coleta de dados, utilizaram-se o Questionário Preliminar de Identificação do Burnout, Síndrome do Esgotamento Profissional (SEP) e Maslach Burnout Inventory (MBI-ED) para educadores, proposto por Maslach e Jackson (1986), traduzido e adaptado ao idioma português para ser utilizado no Brasil por Jbeili (2008). Esse questionário é uma Escala Likert, composto de 20 perguntas que avaliam índices da SB, com cinco opçóes de resposta: 1 para "nunca", 2 para "anualmente", 3 para "mensalmente", 4 para "semanalmente" e 5 para "diariamente". Os indivíduos respondem a perguntas relacionadas às características psicofísicas em relação ao trabalho.

$\mathrm{O}$ questionário classifica a $\mathrm{SB}$ em quatro fases mediante a soma das respostas, que oscila de 0 a 20 pontos. Não há início da SB; de 21 a 40 pontos: fase 1 da $S B$, há possibilidade de desenvolver a síndrome; de 41 a 60 pontos: fase 2 da SB, o indivíduo se encontra em fase inicial da doença; de 61 a 80 pontos: fase 3 da SB, a síndrome começa a se instalar no indivíduo, sendo necessária a ajuda de um profissional para evitar futuro agravamento da doença; de 81 a 100 pontos: fase 4 da SB, fase considerável da SB, sendo possível a reversibilidade por meio de tratamento realizado o quanto antes.

As variáveis dependentes foram coletadas por meio de questionário e categorizadas da seguinte forma: modalidade de ensino (Educação Infantil, Ensino Fundamental anos iniciais, Ensino Fundamental anos finais e Ensino Médio), tempo na docência (menos de 1 ano, de 1 a 5 anos, de 6 a 10 anos e de 11 anos ou mais), trabalha em outra escola pública (sim e não), vínculo empregatício (efetivo, contratado e designado), satisfação com a remuneração (sim e não), jornada semanal na docência (20 horas, 40 horas,
60 horas ou mais de 60 horas), possui algum tipo de doença (sim e não).

Os questionários foram entregues aos professores durante os horários de aula e em cursos de capacitação das escolas públicas estaduais da Jurisdição da Superintendência Estadual de Ensino do município de Januária (MG), em 2013, sendo devolvidos diretamente às pesquisadoras.

Os procedimentos utilizados para a análise estatística foram os recursos da estatística descritiva com frequência, média e desvio-padrão. Para verificar a associação entre $\mathrm{SB}$ e fatores associados, utilizaram-se o teste qui-quadrado $\left(\mathrm{x}^{2}\right)$ e o pacote estatístico Statistical Package for the Social Science (SPSS), versão $20.0^{1}$. O projeto da pesquisa foi aprovado pelo Comitê de Ética em Pesquisa da Universidade Estadual de Montes Claros (Unimontes), por meio do Parecer Consubstanciado n ${ }^{\circ} 152.343$, em 23 de novembro de 2012.

\section{Resultados}

A Tabela 1 apresenta a classificação da amostra de acordo com as modalidades de ensino. Dos 462 professores investigados, registrou-se que 17 (3,7\%) eram professores de Educação Infantil que atendem alunos com idades de quatro e cinco anos; 90 (19,5\%), do Ensino Fundamental - anos iniciais do primeiro ao quinto ano; 251 (54,3\%), do Ensino Fundamental - anos finais do sexto ao nono ano; e 104 (22,5\%), do Ensino Médio.

A Tabela 2 apresenta a classificação dos professores no escore da SB: $12 \%$ dos professores foram classificados na fase 1 , em que há possibilidade de se desenvolver a SB; $59,3 \%$ encontravam-se na fase 2 , fase inicial da doença; os dados apresentados nas duas primeiras fases indicam que é preciso fazer intervenções que possam melhorar as condiçôes de trabalho dos professores, evitando agravamento da SB; $24,2 \%$ estavam na fase 3 e $4,7 \%$, na fase 4 ; aqueles que se encontram nessas fases necessitam de tratamento.

Tabela 1. Descrição da amostra de docentes de acordo com as modalidades de ensino.

\begin{tabular}{lcc}
\hline \multicolumn{1}{c}{ Modalidade de ensino } & $\mathbf{N}$ & $\mathbf{\%}$ \\
\hline Educação Infantil & 17 & 3,7 \\
Ensino Fundamental (AI) & 90 & 19,5 \\
Ensino Fundamental (AF) & 251 & 54,3 \\
Ensino Médio & 104 & 22,5 \\
Total & $\mathbf{4 6 2}$ & $\mathbf{1 0 0 , 0 \%}$ \\
\hline
\end{tabular}

AI: anos iniciais; AF: anos finais. 
$\mathrm{Na}$ Tabela 3, foi utilizado o teste qui-quadrado para verificar se existe associação entre a variável $\mathrm{SB}$ e os fatores associados. Os resultados apresentados permitiram identificar a existência de associação significativa nas seguintes variáveis: vínculo empregatício; satisfação com a remuneração; possui algum tipo de doença. Outra variável que merece atenção é a jornada semanal de trabalho, pois apresentou um valor aproximado de associação com a variável SB. Observou-se que o cargo do professor interfere na percepção em relação ao trabalho. Professores efetivados apresentaram maiores prevalências na fase 4 e professores contratados, na fase 2. Professores insatisfeitos com a remuneraçáo e algum tipo de

Tabela 2. Distribuição dos professores de acordo com as fases da SB.

\begin{tabular}{lcrrrr}
\hline \multirow{2}{*}{ Fase da SEP } & \multicolumn{1}{c}{ Fase 1 } & \multicolumn{1}{c}{ Fase 2 } & \multicolumn{1}{c}{ Fase 3 } & \multicolumn{1}{c}{ Fase 4 } & \multirow{2}{*}{ Total } \\
\cline { 2 - 5 } \multicolumn{1}{c}{$\mathbf{5 7}(\mathbf{1 2 , 3 \% )}$} & $\mathbf{2 7 1 ( 5 8 , 6 \% )}$ & $\mathbf{1 1 2}(\mathbf{2 4 , 2} \%)$ & $\mathbf{2 2}(\mathbf{4 , 7 \% )}$ & \\
\hline Educação Infantil & $01(5,9 \%)$ & $11(64,7 \%)$ & $04(23,5 \%)$ & $01(5,9 \%)$ & $\mathbf{1 7}$ \\
Ensino FAI & $19(21,1 \%)$ & $51(56,6 \%)$ & $16(17,8 \%)$ & $04(4,4 \%)$ & $\mathbf{9 0}$ \\
Ensino FAF & $26(10,3 \%)$ & $152(60,5 \%)$ & $62(24,7 \%)$ & $11(4,4 \%)$ & $\mathbf{2 5 1}$ \\
Ensino Médio & $11(10,6 \%)$ & $57(54,8 \%)$ & $30(28,8 \%)$ & $06(5,7 \%)$ & $\mathbf{1 0 4}$ \\
Total & $57(12,3 \%)$ & $271(58,6 \%)$ & $112(24,2 \%)$ & $22(4,7 \%)$ & $\mathbf{4 6 2}$ \\
\hline
\end{tabular}

FAI: Fundamental anos iniciais; FAF: Fundamental anos finais.

Tabela 3. Teste qui-quadrado da variável SB com as variáveis independentes.

\begin{tabular}{|c|c|c|c|c|c|c|c|}
\hline Variável & $\begin{array}{c}\text { Fase } 1 \\
\%\end{array}$ & $\begin{array}{c}\text { Fase } 2 \\
\%\end{array}$ & $\begin{array}{c}\text { Fase } 3 \\
\%\end{array}$ & $\begin{array}{c}\text { Fase } 4 \\
\%\end{array}$ & Total & $\mathbf{X}^{2}$ & P-valor \\
\hline \multicolumn{8}{|l|}{ Sexo } \\
\hline Masculino & 13,9 & 56,6 & 23,8 & 5,7 & 100 & 0,814 & 0,846 \\
\hline Feminino & 11,8 & 59,4 & 24,4 & 4,4 & 100 & & \\
\hline \multicolumn{8}{|l|}{ Modalidade de ensino } \\
\hline Educação Infantil & 5,9 & 64,7 & 23,5 & 5,9 & 100 & 10,679 & 0,298 \\
\hline Ensino FAI & 21,1 & 56,7 & 17,8 & 4,4 & 100 & & \\
\hline Ensino FAF & 10,4 & 60,6 & 24,7 & 4,4 & 100 & & \\
\hline Ensino Médio & 10,6 & 54,8 & 28,8 & 5,8 & 100 & & \\
\hline \multicolumn{8}{|c|}{ Quanto tempo de docência } \\
\hline Menos de 1 ano & 22,7 & 63,6 & 13,6 & 0 & 100 & 13,521 & 0,140 \\
\hline 1 a 5 anos & 15,6 & 65,6 & 16,7 & 2,2 & 100 & & \\
\hline 6 a 10 anos & 11,7 & 59,4 & 25 & 3,9 & 100 & & \\
\hline 11 ou mais & 10,4 & 55,0 & 27,9 & 6,8 & 100 & & \\
\hline \multicolumn{8}{|c|}{ Trabalha em outra escola pública } \\
\hline Sim & 10,7 & 60,7 & 23,8 & 4,8 & 100 & 0,775 & 0,856 \\
\hline Não & 13,3 & $57,5 \%$ & 24,5 & 4,8 & 100 & & \\
\hline \multicolumn{8}{|l|}{ Vínculo empregatício } \\
\hline Efetivo & 14,1 & 54,2 & 27,1 & 4,5 & 100 & 20,843 & 0,013 \\
\hline Contratado & 6,5 & 87,1 & 3,2 & 3,2 & 100 & & \\
\hline Designado & 14,3 & 62,2 & 21,8 & 1,7 & 100 & & \\
\hline Efetivado & 9,6 & 54,8 & 27,4 & 8,1 & 100 & & \\
\hline \multicolumn{8}{|c|}{ Está satisfeito com a remuneração } \\
\hline $\operatorname{Sim}$ & 32,8 & 58,6 & 8,6 & 0 & $100 \%$ & 32,254 & 0,000 \\
\hline Não & 9,4 & 58,7 & 26,5 & 5,4 & $100 \%$ & & \\
\hline \multicolumn{8}{|c|}{ Jornada semanal na docência } \\
\hline 20 horas & 13,1 & 63,4 & 20,8 & 2,7 & 100 & 15,558 & 0,077 \\
\hline 40 horas & 10,9 & 57,3 & 26,8 & 5,0 & 100 & & \\
\hline 60 horas & 21,7 & 34,8 & 30,4 & 13,0 & 100 & & \\
\hline Mais de 60 & 15,4 & 53,8 & 15,4 & 15,4 & 100 & & \\
\hline \multicolumn{8}{|c|}{ Possui algum tipo de doença } \\
\hline Sim & 6,5 & 52,2 & 32,6 & 8,7 & 100 & 11,023 & 0,012 \\
\hline Não & 13,8 & 60,3 & 22,2 & 3,8 & 100 & & \\
\hline
\end{tabular}

FAI: Fundamental anos iniciais; FAF: Fundamental anos finais. 
doença apresentaram-se, em maior proporção, nas fases 3 e 4 .

\section{Discussão}

O presente estudo verificou a prevalência da SB em professores da rede pública estadual de ensino e fatores associados. O principal achado foi que aproximadamente $30 \%$ dos professores apresentaram SB (fases 3 e 4). Além do mais, a baixa remuneração na funçáo e o fato de ser professor efetivado na carreira profissional são fatores associados a maiores prevalências da SB.

Os dados obtidos nesta pesquisa apontaram que $24 \%$ encontram-se na fase 3 e $4,7 \%$, na fase 4 . Esses resultados são semelhantes aos encontrados no estudo realizado nas escolas da região metropolitana de Porto Alegre (RS) por Carlotto (2011), que verificou $28,9 \%$ de professores com princípio de SB.

Resultados mais expressivos foram encontrados em um estudo realizado por Batista et al. (2010) com professores de João Pessoa (PB), em que 43,4\% deles apresentaram fase de risco na classificação da síndrome Outra investigação, desenvolvida por Benevides-Pereira et al. (2008), analisando a SB em professores paranaenses também evidenciou maiores resultados nas fases de risco, com 43,3\%.

Corroborando com os estudos citados, um estudo realizado por Codo (1999), que investigou 52 mil professores da rede pública de ensino nos 24 estados da Federação Brasileira, concluiu que $48 \%$ manifestaram algum sintoma de burnout, o que, segundo o autor, pode levar à falência da educação.

Mesmo sendo uma profissão em que a maioria dos estudos aponta para a prevalência da $\mathrm{SB}$, o estudo realizado com professores de escolas públicas e privadas de Viçosa (MG) não identificou SB (FERREIRA; SANTOS; RIGOLON, 2014).

Baixa predisposição à SB foi encontrada em um estudo realizado com professores da Universidade de Parnaíba (PI), cujo dados revelaram que 34,5\% deles apresentaram nível baixo de exaustão; em contrapartida, 32,75\% apresentaram níveis de médio a alto. A respeito da realização profissional, $35,4 \%$ apontaram um nível baixo; 32,7\%, alto; e $31,9 \%$, médio. Para a despersonalização, 38,9\% demonstraram ter baixo índice; $31,9 \%$, médio; e 29,2\%, alto. Embora os resultados apresentados indiquem na dimensão exaustáo a existência de esgotamento, não revelaram alta predisposição da SB (SILVA et al., 2014).

A profissão docente expõe-se a diversas fontes geradoras de tensão, como elevada carga horária de trabalho, elevado número de alunos por sala de aula, estrutura física inadequada, poucos trabalhos pedagógicos em equipe, pouca participação da família no que concerne ao acompanhamento do desenvolvimento escolar de seus filhos, baixos salários, desvalorização da profissáo, excesso de cobrança, uso inadequado das redes sociais na internet pelos alunos no horário de aula, desrespeito, desinteresse, ameaças dos alunos para com os professores.

O desinteresse dos alunos da rede pública, somado aos baixos salários, acarreta sentimento de insatisfaçáo dos docentes, dificultando que seus esforços sejam convertidos em aprendizado. Entretanto, na rede privada, os docentes tendem a questionar o excesso de cobranças, mesmo em condiçóes nas quais sua produtividade é adequada (FERREIRA; SANTOS; RIGOLON, 2014).

Outro fator que prejudica a profissáo docente são políticas pedagógicas e políticas públicas que norteiam a educação, especificamente no estado de Minas Gerais, que efetivou os servidores públicos sem concurso por meio da Lei $n^{\circ} 100 / 2007$, que deixa o professor na incerteza do cargo. A Lei Complementar $\mathrm{n}^{\circ}$ 100/2007 (MINAS GERAIS, 2007) foi instituída em 5 de novembro de 2007 e regulariza a situação jurídica criada pelos incisos IV e $\mathrm{V}$ do Artigo 7 da referida lei, que acolheu os docentes no sistema previdenciário para efeito de aposentadoria e concedeu o voto e a participação em todos os cargos existentes na instituição. Os professores não se tornaram efetivos, e sim efetivados, pois a funçáo de efetivo ocorre somente por meio de concurso público (BRASIL,1988).

A forma como os professores lidam com esse cotidiano potencialmente adverso pode repercutir de maneira negativa, gerando problemas físicos e psicológicos. Dentre as problemáticas que mais acometem os docentes atualmente, temos a SB, que, neste estudo, apresentou alta prevalência entre os professores.

Ferreira, Santos e Rigolon (2014) destacam que a insatisfação profissional e as condições inadequadas de trabalho podem desencadear alteraçôes no bem-estar físico ou psicológico desses profissionais, deixando-os mais propícios a desenvolver a burnout e levando-os a se afastarem de seus postos de trabalho.

Os resultados apresentados no presente estudo são preocupantes, pois apontaram um índice alto de predisposição à $\mathrm{SB}$, merecendo atenção, pois os professores continuam em atividade e náo existe uma política de saúde para atender essa população.

Segundo Maslach e Goldberg (1998), apesar de que muitas pessoas possam deixar o trabalho 
em consequência do burnout, outras podem permanecer exercendo suas funçóes, ainda que trabalhando muito abaixo do seu potencial laboral e apresentando problemas na qualidade do trabalho desenvolvido. Ainda nessa linha de pensamento, tais resultados carecem de atenção particular, uma vez que os professores identificados e sinalizados com a SB estão em exercício funcional, provavelmente agravando seu quadro com prejuízos para a qualidade do seu trabalho e com consequências na relação ensino-aprendizagem do aluno conforme referem (GIL-MONTE; CARLOTTO; CÂMARA, 2011).

Os resultados do presente estudo apontaram que dentre os docentes que se encontram na fase $4 \mathrm{da}$ SB, $8,1 \%$ foram efetivados e apresentaram maior risco da $\mathrm{SB}$ devido à insegurança do cargo. Nota-se uma carência de estudos que avaliem a insatisfação e o desenvolvimento da SB nessa população específica, impossibilitando uma discussão mais abrangente.

Outro fator que apresentou maior prevalência na SB foi a baixa remuneração, que muitas vezes leva os professores a exercer dois cargos, trabalhando em turnos diferentes, podendo ter um elevado desgaste mental e físico. A questáo salarial é fator desencadeador de vários problemas, em razão do qual, principalmente os docentes do ensino público, tendem a realizar dupla ou tripla jornada de trabalho para obter uma melhor remuneraçáo, tendo pouco tempo para descansar e se dedicar à família. É comum esses profissionais apresentarem sintomas de estresse, depressão e ansiedade, característicos da SB (FERREIRA; SANTOS; RIGOLON, 2014).

$\mathrm{O}$ volume e a sobrecarga de trabalho têm sido as variáveis mais predisponentes à SB (CARLOTO, 2011), ou seja, quando a quantidade e a qualidade das demandas de trabalho ultrapassam a capacidade de desempenho pessoal, o indivíduo tenderá a adoecer. No estudo de Codo (1999), os achados indicaram que professores de primeiro e segundo graus de escolas públicas sofreram elevados níveis de estresse em razão dos menores salários e de piores condições de trabalho

Na presente pesquisa, destacam-se como limitaçóes a resistência de diretores em permitir a realização da pesquisa em algumas escolas e a resistência de alguns professores em participar da pesquisa ou responder aos questionários.

Os resultados encontrados neste estudo apontam que há um alto indíce de professores das escolas da Jurisdição da SEE de Januária (MG) com SB. Assim, é preciso propor intervençôes nas escolas com $\mathrm{o}$ intuito de orientar e incentivar seus dirigentes e professores sobre a importância de se planejar as atividades pedagógicas e enfrentar o burnout, de forma a envolver os docentes, as instituiçóes escolares e os gestores. Além disso, os achados deste estudo podem ser importantes para nortear as diretrizes de políticas públicas de promoção de saúde dos docentes.

\section{Conclusão}

Os resultados apresentados nesse estudo permitiram identificar alta prevalência da $\mathrm{SB}$, que atinge um a cada três professores do ensino estadual público e gratuito. Esse índice é preocupante, pois os professores continuam em atividade educacional sem intervenção que possa melhorar o quadro, podendo, assim, levar à falência do processo ensino-aprendizagem na Jurisdição da SEE de Januária.

Ser professor efetivo ou efetivado, com dedicação de 40 horas semanais ou mais, foi requisito para se evidenciar SB em seu grau mais agudo. Baixos salários conduzem os professores a trabalhar em dois ou três turnos, com várias turmas diferentes, favorecendo a alta predisposição à SB.

Intervençôes com suporte emocional são recomendáveis para os professores, com o intuito de prevenir e utilizar estratégias de enfrentamento das consequências causadas pela SB.

\section{Referências}

BATISTA, J. B. V. et al. Prevalência da Síndrome de Burnout e fatores sociodemográficos e laborais em professores de escolas municipais da cidade de João Pessoa. Revista Brasileira de Epidemiologia, São Paulo, v. 13, n. 3, p. 502-512, 2010.

BENEVIDES-PEREIRA, A. M. T. et al. O trabalho docente e o Burnout: um estudo em professores paranaenses. Curitiba: PUCPR, 2008. Disponível em: <http:// www.pucpr.edu.br/eventos/educere/educere2008/anais/ pdf/550_775.pdf>. Acesso em: 26 set 2016.

BOTH, J. Bem estar do trabalhador docente em Educaçāo Física da regiäo sul do Brasil. 2011. 248 f. Tese (Doutorado em Educação Física) - Universidade Federal de Santa Catarina, Florianópolis, 2011.

BRASIL. Constituiçáo (1988). Emenda constitucional $\mathrm{n}^{\circ}$ 9, de 9 de novembro de 1995. Lex: legislaçáo federal e marginalia. São Paulo, v. 59, p. 1966, 1995.

CARLOTTO, M. S. Síndrome de Burnout em professores: prevalência e fatores associados. Psicologia: Teoria e Pesquisa, Brasília, v. 27, n. 4, p. 403-410, 2011.

CODO, W. Educaçāo: carinho e trabalho: Burnout, a síndrome da desistência do educador, que pode levar à falência da Educação. Petrópolis: Vozes, 1999.

FARBER, B. A. Crisis in education: stress and burnout in the American teacher. São Francisco: Jossey-Bass Inc, 1991. 
FERREIRA, A. A. E.; SANTOS, D. E.; RIGOLON, R. G. Avaliação comparativa dos sintomas da síndrome de burnout em professores de escolas públicas e privadas. Revista Brasileira de Educação, Viçosa, v. 19, n. 59, p. 987-1002, 2014.

GIL-MONTE, P. R. El sindrome de quemarse por el trabajo (Burnout): una enfermidad laboral en la sociedad del bienestar. Madrid: Pirâmide, 2005.

GIL-MONTE, P. R.; CARLOTTO, M. S.; CÂMARA, S. Prevalence of burnout in a sample of Brazilian teachers. The European Journal of Psychiatry, Zarangoza, v. 25, n. 4, p. 205-212, 2011.

HEUS, P.; DIEKSTRA, R. F. W. Do you teachers burnout more easily? A comparison of teachers with other social professions on work stress and burnout symptoms. In: VANDERBERGUE, R.; HUBERMAN, M. A. (Ed.). Understanding and preventing teacher burnout: a source book of international practice and research. Cambridge: Cambridge University Press, 1999. p. 269-284.

JBEILI, C. Questionário JBEILI para identificação preliminar da Burnout. Montes Claros: UNICEAD, 2008. Disponível em: < http://www.chafic.com.br/index_arquivos/Question\%E1rio\%20Jbeili\%20para\%20 identifica\%E7\%E3o\%20da\%20burnout.pdf>. Acesso em: 08 fev. 2011.

KOVESS-MASFÉTY, V. et al. Do teachers have more health problems? Results from a French cross-sectional survey. BMC Public Health, London, v. 101, n. 6, p. $1-13,2006$.

MASLACH, C. Burnout: the cost of caring. Cambridge: Malor Books, 2003.

MASLACH, C.; GOLDBERG, J. Prevention of burnout: news perspectives. Applied \& Preventive Psychology, California, v. 7, p. 63-74, 1998.
MASLACH, C.; JACKSON, S. E. Maslach Burnout inventory. Palo Alto: Consulting Psychologist Press, 1986.

MASLACH, C.; SCHAUFELI, W. B.; LEITER, M. P. Job burnout. Annual Review Psychology, California, n. 52, p. 397-422, 2001.

MINAS GERAIS. Governo do Estado. Lei Complementar $n^{\circ} 100$ de 5 de novembro de 2007. Institui a Unidade de Gestão Previdenciária Integrada - Ugeprevi - do Regime Próprio de Previdência dos Servidores Públicos do Estado de Minas Gerais e do Regime Próprio de Previdência dos Militares do Estado de Minas Gerais e o Conselho Estadual de Previdência - Ceprev -, altera a Lei Complementar no 64, de 25 de março de 2002, e dá outras providências. Diário Oficial dos Poderes do Estado, Belo Horizonte, 5 nov. 2007.

NACARATO, A. M.; VARANI, A.; CARVALHO, V. O cotidiano do trabalho docente: palco, bastidores e trabalho invisível... abrindo as cortinas. In: GERALDI, C. M. G.; FIORENTINA, D.; PEREIRA, E. M. A. (Org.). Cartografias do trabalho docente. Campinas: Mercado de Letras, 2000. p. 45-79.

SEGURA, O. Burnout: concepts and implications affecting public health. Biomedica, Bogotá, v. 34, n. 4, p. 535-545, 2014.

SILVA, M. F. M. et al. Estudo avaliativo da predisposição à síndrome de burnout em professores de uma Universidade de Parnaíba-PI. Revista Psicologia e Saúde, Campo Grande, v. 6, n. 2, p. 28-37, 2014.

SILVEIRA, K. A. et al. Estresse e enfrentamento em professores uma análise da literatura. Educação em Revista, Belo Horizonte, v. 30, n. 4, p. 15-36, 2014.

SOUSA, I. F.; MENDONÇA, H. Burnout em professores universitários: impacto de percepçóes de justiça e comprometimento afetivo. Psicologia: Teoria e Pesquisa, Brasília, v. 25, n. 4, p. 499-508, 2009.

\section{Contribuição dos Autores}

Adelson: Coordenação, Organização do Estudo e redação do texto. Maria de Fátima: Concepção do texto e organização de fontes. Celina Aparecida: Análises dos dados. Izulina e Karoline: Redação do texto e pesquisa de campo. Diego Augusto e Edio Luiz: Análise dos dados e Revisão. Todos os autores aprovaram a versão final do texto.

\section{Notas}

${ }^{1}$ Licença: 4B6MINO86Z4LZV9AA7GHEC89P5TRNTOHAA3XKX5YW7GM2SWHCCTAFYBL3B3IKPM M7I9N3MSTBXOO8VPKXZHSEXGST8 